\section{MS32-P14 Structural characterization of tenofovir disoproxil fumarate Form I using X-ray and electron diffraction and a study of its conversion to related solid forms}

Veronika Sládková ${ }^{1}$, Eliška Skořepová ${ }^{1}$, Jan Čejka ${ }^{1}$, Ondřej Dammer $^{2}$, Bohumil Kratochvíl ${ }^{1}$, Petr Brázda ${ }^{3}$, Jan Rohlíček

1. Department of Solid State Chemistry, University of Chemistry and Technology, Prague, Technicka 5, 16628, Prague 6, Czech Republic

2. Department of Solid State Development, Zentiva k.s., U Kabelovny 130, 102 37, Prague, Czech Republic

3. Institute of Physics AS CR, v.v.i., Na Slovance 2, 18221 Prague

8, Czech Republic

email: sladkovv@vscht.cz

Tenofovir disoproxil fumarate (TDF) is an orally delivered pharmaceutical compound used for the treatment of HIV and chronic hepatitis, which acts as an inhibitor of nucleotide reverse transcriptase. There are many solid forms of TDF described in patent literature; two of them we identified in drug products: Form I and Form A. It seems that during formulation the active pharmaceutical ingredient (API) undergoes partial to total conversion of TDF Form I to TDF Form A. The aim of the study was to propose a formulation of tablet containing pure TDF Form I. However, we observed that TDF Form I converted either to TDF Form A or recently described TDF Form I-1. We investigated, when and why did the conversion occur and whether the conversion could be avoided, and how. The influence of $\mathrm{pH}$ and possible interaction with excipients were studied. The conditions enabling using wet granulation in technology while preventing the undesired conversion were found. The stabilization was achieved either by replacement of used disintegrants or $\mathrm{pH}$ adjustment by acid addition to the current composition of formulation.

We also found that TDF Form I underwent the same non-reversible phase transformation to TDF Form I-1 both upon heating, as well as upon exposure to humidity. The phenomenon was observed by temperature resolved X-ray powder diffraction (XRPD), solid state NMR spectroscopy and DSC.

As neither structure of TDF Form I nor of TDF Form I-1 has been determined, we focused on structure solution by combining high quality XRPD, synchrotron single crystal XRD and electron diffraction. We were successful in indexing of the powder of TDF Form I, which was in agreement with the indexing of the microcrystals measured on synchrotron. Moreover, a structure of another tenofovir compound - tenofovir disoproxil phosphate, was successfully determined from single crystal XRD data.

Acknowledgment: This work was supported by the Grant Agency of Czech Republic, Grant no. $106 / 16 / 10035 \mathrm{~S}$ and received financial support from specific university research (MSMT No 20/2016). We acknowledge the ESRF for provision of synchrotron radiation facilities and we would like to thank J. Wright for assistance in using beamline ID11.

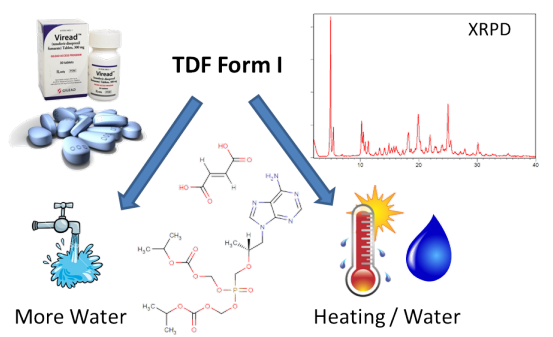

TDF Form A

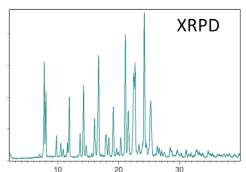

Figure 1. TDF Form I converted either to TDF Form A upon slurrying in water or to recently described TDF Form I-1 upon heating or exposure to water.

Keywords: pharmaceuticals, phase transformation, electron diffraction, $\mathrm{x}$-ray powder diffraction, indexing 